\title{
Temporal and spatial changes of cells positive for stem-like markers in different compartments and stages of human colorectal adenoma-carcinoma sequence
}

\author{
Guanglin Cui ${ }^{1}{ }^{12}$, Gang $\mathrm{Xu}^{1}$, Li Zhu ${ }^{1}$, Zhigang Pang ${ }^{1}$, Wei Zheng ${ }^{1}$, Zhenfeng $\mathrm{Li}^{1}$ and \\ Aping Yuan ${ }^{1}$ \\ ${ }^{1}$ Research Group of Gastrointestinal Diseases, the Second Affiliated Hospital of Zhengzhou University, Zhengzhou, Henan, \\ China \\ ${ }^{2}$ Faculty of Health, Nord University, Levanger, Norway
}

Correspondence to: Guanglin Cui, email: guanglin.cui@yahoo.com

Keywords: stem-like marker, tumorigenesis, colorectum

Received: October 13, $2016 \quad$ Accepted: April 11, $2017 \quad$ Published: April 21, 2017

Copyright: Cui et al. This is an open-access article distributed under the terms of the Creative Commons Attribution License 3.0 (CC BY 3.0), which permits unrestricted use, distribution, and reproduction in any medium, provided the original author and source are credited.

\section{ABSTRACT}

Considerable evidence supports the idea that stem-like cells may play an essential role during the development of colorectal cancer (CRC). To accomplish this aim, we use immunohistochemistry (IHC) and double IHC with different potential stem-like markers, anti-musashi (Msi), anti-CD133, anti- LGR5 and anti-ALDH1 to examine the presentation of stem-like cells in different compartments including adenoma/CRC epithelium, transitional crypts and tumor stroma in colorectal adenoma and CRC. The results showed that cells positive for stem-like markers were remarkably increased in number and frequently observed in the adenoma/CRC epithelium, transitional crypts and tumor stroma. Notably, the population of cells positive for stem-liker markers was expanded from the base to the middle part of the transitional crypt in both adenoma and CRC tissues, reflecting that stem-like cells are likely involved in the process of colorectal tumorigenesis. Counting results showed that the grading scores of cells positive for LGR5 and ALDH1 in the adenoma/CRC epithelium were significantly increased relative with the control epithelium, and associated with the degree of dysplasia in the adenoma and node involvement in the CRC (all $P<0.05$ ). In addition, the density of cells positive for stem-like markers in the adenomatous/cancerous stroma was also increased and paralleled an increase in the density of proliferative stromal cells labeled by PCNA, which were primarily identified as vimentin positive fibroblasts. Our results have revealed a changed temporal and spatial presentation of stem-like markers in different stages of human colorectal adenoma-carcinoma sequence, which might be a hallmark of the adenoma-carcinoma transition.

\section{INTRODUCTION}

Colorectal cancer (CRC) is the fourth most frequent cancer and has a high mortality rate worldwide. Most CRCs, according to the adenoma-carcinoma sequence theory, develop from preformed adenoma polyps. The malignant potential of an adenomatous polyp is associated with its size, dysplastic degree and severity of atypia. This adenoma-carcinoma sequence provides an ideal opportunity to investigate the changes that occur during the development of CRC. Although many molecular, genetic and immunological alterations that are involved in this sequence have been identified [1-3], the exact mechanisms of the adenoma-carcinoma transition are still not fully understood.

Recent studies have provided considerable evidence to support the hypothesis that human tumors are derived from a subpopulation of cells with stem-like cell properties, termed tumor stem cells $[1,2]$. Tumor stemlike cells have the capacity to self-renew, differentiate and drive tumorigenic growth [2]. The existence of tumor stem-like cells in precancerous adenomas as well as in 
CRCs have been described by several groups [2-10]. An association between the tumor stem-like cells and the prognosis of patients with CRC has also been suggested $[11,12]$. During cancer initiation, the changes of tumor stem-like cells from precancerous to cancerous lesions are of particular interest because the alternation of tumor stem-like cells may be one of the hallmarks for colorectal tumorigenesis $[10,13,14]$. The histological identification of stem-like cells in either tumor or non-tumor tissues relies largely on specific markers. Several putative markers i.e., Musashi (Msi), CD44, CD133, leucine-rich repeat-containing G-protein-coupled receptor 5 (LGR5) and aldehyde dehydrogenase 1 (ALDH1) have been used to identify tumor stem-like cells in CRCs [2-9]. However, previous studies have primarily examined the presentation of stem-like markers in established adenomatous/ cancerous epithelium; temporal and spatial variation of stem-like markers in different stages of colorectal adenoma-carcinoma sequence have not been well studied.

Given the above background, we hypothesized that an altered temporal and spatial presentation of stem-like markers could be an important hallmark that reflects the ongoing tumorigenesis in colorectum. In this study, we have therefore used different stem-like markers, Msi, CD133, LGR5 and ALDH1 to investigate the presentation of stem-like cells in the different compartment and histological stages of the colorectal adenoma-carcinoma sequence.

\section{RESULTS}

\section{Stem-like markers in the adenomatous/cancerous epithelium}

The presence of stem-like markers in different stages of the adenoma-carcinoma sequence was evaluated with IHC using Msi, CD133, LGR5 and ALDH1 antibodies (Figure 1). The results showed that in normal controls, a few stem-like marker (Msi, CD133, LGR5 and ALDH1) positive cells were at the base of normal colonic crypts (Figure 1A). In the adenomatous epithelium, the cells positive for stem-like markers were expressed in a patchy distribution pattern on the surface of the adenomatous crypt (Figure 1B), In the cancerous epithelium, the cells positive for stem-like markers were also distributed in a diffused pattern (Figure 1C) or in the surface of CRC epithelium (Figure 1F) as reported in the literature [5].

Because LGR5 and ALDH1 are the two most promising and established stem-like markers in adenoma/ CRC, we examined the immunoreactivity of LGR5 and ALDH1 in the adenoma/CRC epithelium with semiquantitative analysis. The percentage of cells positive for LGR5 and ALDH1 in the adenomatous epithelium was $39.47 \%$ (15/38) and $47.37 \%$ (18/38), respectively. Additionally, the percentage of cells positive for LGR5 and
ALDH1 in the CRC epithelium was 63.33\% (19/30) and $66.67 \%$ (20/30), respectively. The positive rate for LGR5 and ALDH1 in the CRC epithelium were slightly higher than that in the adenomatous epithelium (both $P>0.05$ ).

Then, the grading scores of LGR5 positive and ALDH1 positive cells in the adenomatous/cancerous epithelium were analyzed against clinical pathological parameters in adenomas and CRCs. In the adenoma, the grading scores of LGR5 positive and ALDH1 positive cells were correlated with degree of dysplasia (low-grade dysplasia vs. high-grade dysplasia; LGR5: $1.26 \pm 0.10$ vs. $166 \pm 0.12$; ALDH1: $1.52 \pm 0.11$ vs. $1.98 \pm 0.14$; both $P<0.05$ by the Mann-Whitney test), but not with histological types (data not shown). In the CRC, the grading scores of both LGR5 positive and ALDH1 positive cells were associated with node involvement (node negative vs. node positive: LGR5, $1.44 \pm 0.13$ vs. $2.18 \pm 0.24 ;$ ALDH1, $1.74 \pm 0.13$ vs. $2.40 \pm 0.20$ : both $P<0.05$ by the Mann-Whitney test). Both of LGR5 and ALDH1 grading scores were higher in CRC patients with advanced TNM stage than those with early stage (TNM stage I vs. II vs. III + VI: LGR5, $1.40 \pm 0.17$ vs. $1.43 \pm 0.15$ vs. $2.27 \pm 0.26, P<0.05$; ALDH1, $1.60 \pm 0.19$ vs. $1.83 \pm 0.16$ vs. $2.29 \pm 0.19, P>0.05$; both by the Kruskal-Wallis test).

\section{Changed presentation pattern of cells positive for stem-like markers in the transitional crypts close to adenomas and CRCs}

Notably, in those sections with transitional crypts (6 of 30 adenoma sections, 5 of 30 CRC sections), a changed presentation pattern of cells positive for stemlike markers (Msi, CD133, LGR5 and ALDH1) was observed (see Figure 2). The location of cells positive for stem-like markers Msi, CD133, LGR5 and ALDH1 was expanded, often from the base to the middle part and occasionally to the top of the transitional crypt close to adenomas and CRCs (transitional crypt in adenomas, see Figure 2A, 2C, 2E and 2G; transitional crypt in CRCs, see Figure 2B, 2D, 2F and 2H). Those observations strongly suggested that expanding pathway of stem-like cells is from the base to the top during the development of adenomas and CRCs.

\section{Increased mesenchymal cells positive for stem- like markers in adenomatous/cancerous tumor stroma}

Mesenchymal stem-like cells resided in the tumor stroma play a critical role in forming a supportive environment for cancer growth and metastasis [21, 22]. In normal controls, the cells positive for stem-like markers could be observed in the lamina propria. The cells positive for stem-like markers Msi, CD133, LGR5 and ALDH1 were diffusely distributed, and the density of positive 
cells in lamina propria was higher than that in the crypt (Figure 3A, 3D, 3G and 3J). In adenoma tissues, the cells positive for stem-like markers were diffusely distributed in the tumor stroma between adenomatous epithelium (Figure 3B, 3E, 3H and 3J). In CRC tissue, the distribution pattern of cells positive for stem-like markers in tumor stroma (see Figure 3C, 3F, 3I and 3L) was very similar to that in adenomas. However, counting results showed that the densities of cells positive for stem-like markers LGR5 (Figure 4A) and ALDH1 (Figure 4B) in CRC tumor stroma were slightly higher than that in adenomas.

Then, the grading scores of LGR5 positive and ALDH1 positive cells in the adenomatous/cancerous stroma were analyzed against clinical pathological parameters. In the adenoma stroma, the grading scores of LGR5 positive and ALDH1 positive cells were not correlated with degree of dysplasia or histological types (data not shown). In the CRC stroma, the grading scores of LGR5 positive and ALDH1 positive cells were not associated with TNM stages (NM stage I vs. II vs. III + VI: LGR5, $16.86 \pm 1.87$ vs. $15.18 \pm 1.52$ vs. $6.11 \pm 2.45, P>0.05 ;$ ALDH1, $18.71 \pm 2.69$ vs. $20.36 \pm 2.16$ vs. $18.78 \pm 2.15, P>0.05$; both by the Kruskal-Wallis test). However, we have found that although the difference did not reach statistical significance, the density grading scores were higher in CRC patients with node involvement than those without (node negative vs. node positive: LGR5, 14.89 \pm 1.20

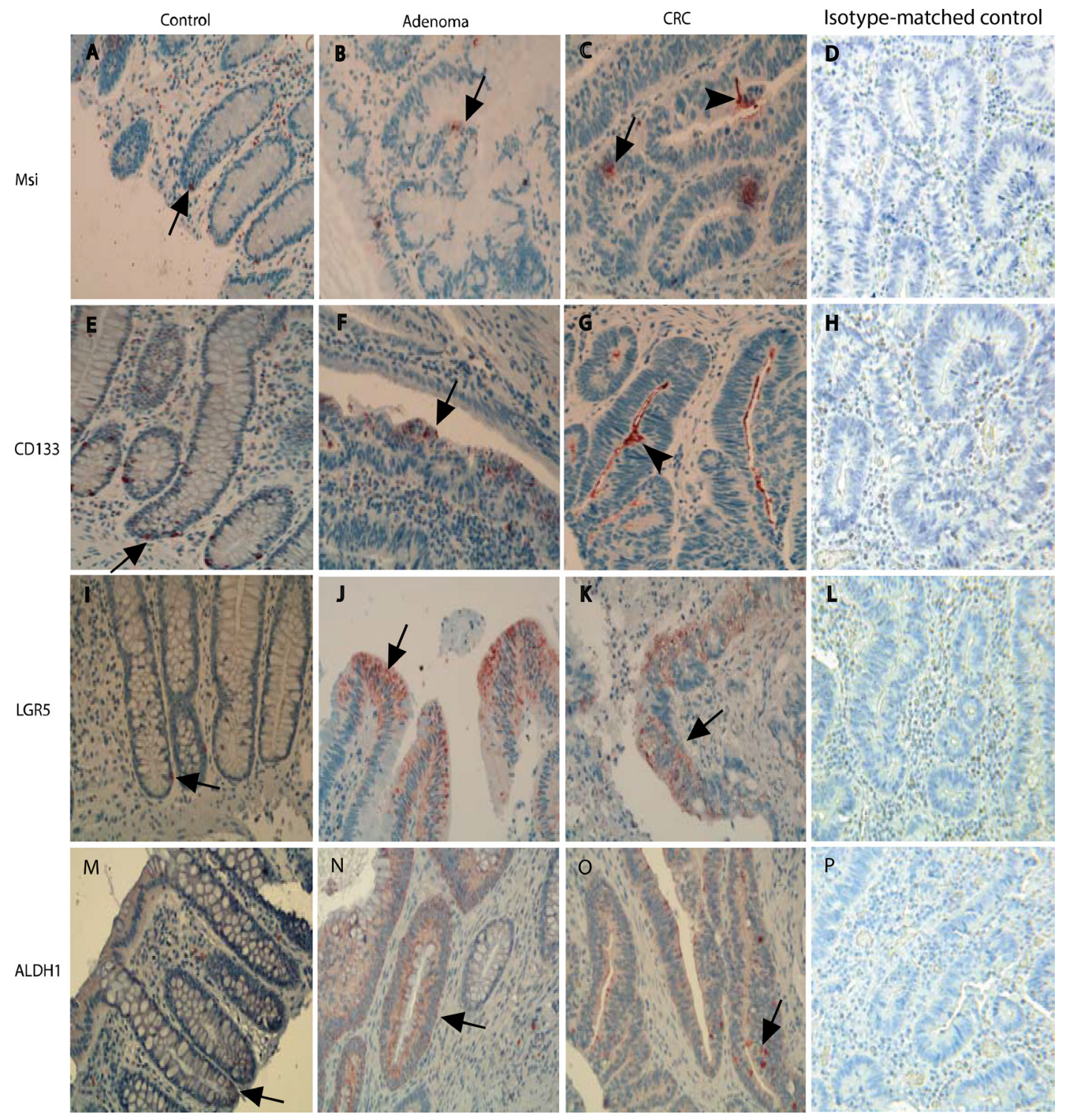

Figure 1: The presentation of stem-like markers Msi, CD133, LGR5 and ALDH1 in different stages of the adenomacarcinomas evaluated with IHCs. In normal controls, a few cells positive for stem-like markers were shown at the base of normal colonic crypts (Figure 1A, 1E, 1I and 1M). In the adenomatous epithelium, the cells positive for stem-like markers were expressed as a patchy destruction pattern in the surface of adenomatous crypt (Figure $\mathbf{1 B}, \mathbf{1 F}, \mathbf{1 J}$ and $\mathbf{1 N}$ ), In the CRC epithelium, the cells positive for stem-like markers were also distributed in a diffusely pattern (Figure 1C, 1G, 1K and 10). In sections with isotype-matched negative control antibodies staining (Figure 1D, 1H, 1L and 1P), no any positive cells were found. 

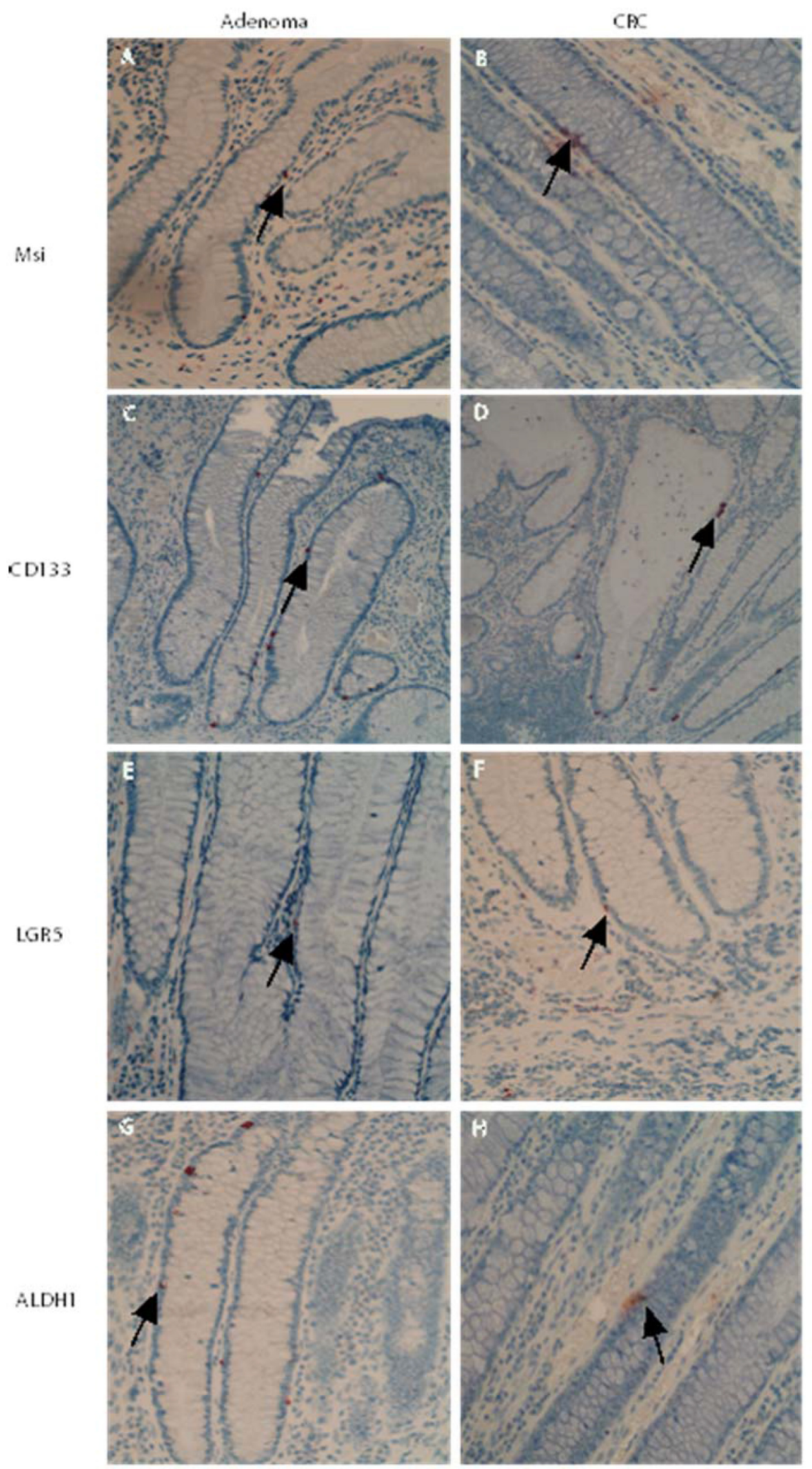

Figure 2: Changed presentation pattern of stem-like markers in the transitional crypts close to adenomas and CRCs. In transitional crypts close to adenomas and CRCs, a changed presentation pattern of stem-like markers was observed. The populations of cells positive for stem-like markers Msi, CD133, LGR5 and ALDH1 were expanded, often from the base to the middle part, or even higher part of the transitional crypt (transitional crypt in adenomas, see Figure 2A, 2C, 2E and 2G; transitional crypt in CRCs, see Figure 2B, 2D, 2F and 2H). 
vs. $18.00 \pm 2.19 ;$ ALDH1, $18.00 \pm 1.37$ vs. $22.22 \pm 2.59$; both $P<0.05$ by the Mann-Whitney test).

\section{Increase in cells positive for stem-like markers in the adenomatous/cancerous stroma was accompanied by an increase in PCNA-positive proliferative cells that consisted primarily of fibroblasts}

Tumor stroma and contributes to the cancer initiation and progression [23]. We therefore investigated proliferation activity of stromal cells in the
adenomatous/CRC stroma. Along with the increase in cells positive for stem-like markers in the tumor stroma, the number of proliferative cells in the adenomatous/ cancerous tumor stroma was also significantly increased. The proliferation rate in the tumor stromal cells was evaluated with PCNA IHC (Figure 5A-5C). The results showed that the proliferation rate increased in both adenomatous and cancerous tumor stromal cells compared to controls (Figure 5G) (both $P<0.0001$ by the Mann-Whitney test). No significant increase in proliferation rate was observed between adenoma and CRC stroma $(P>0.05)$.
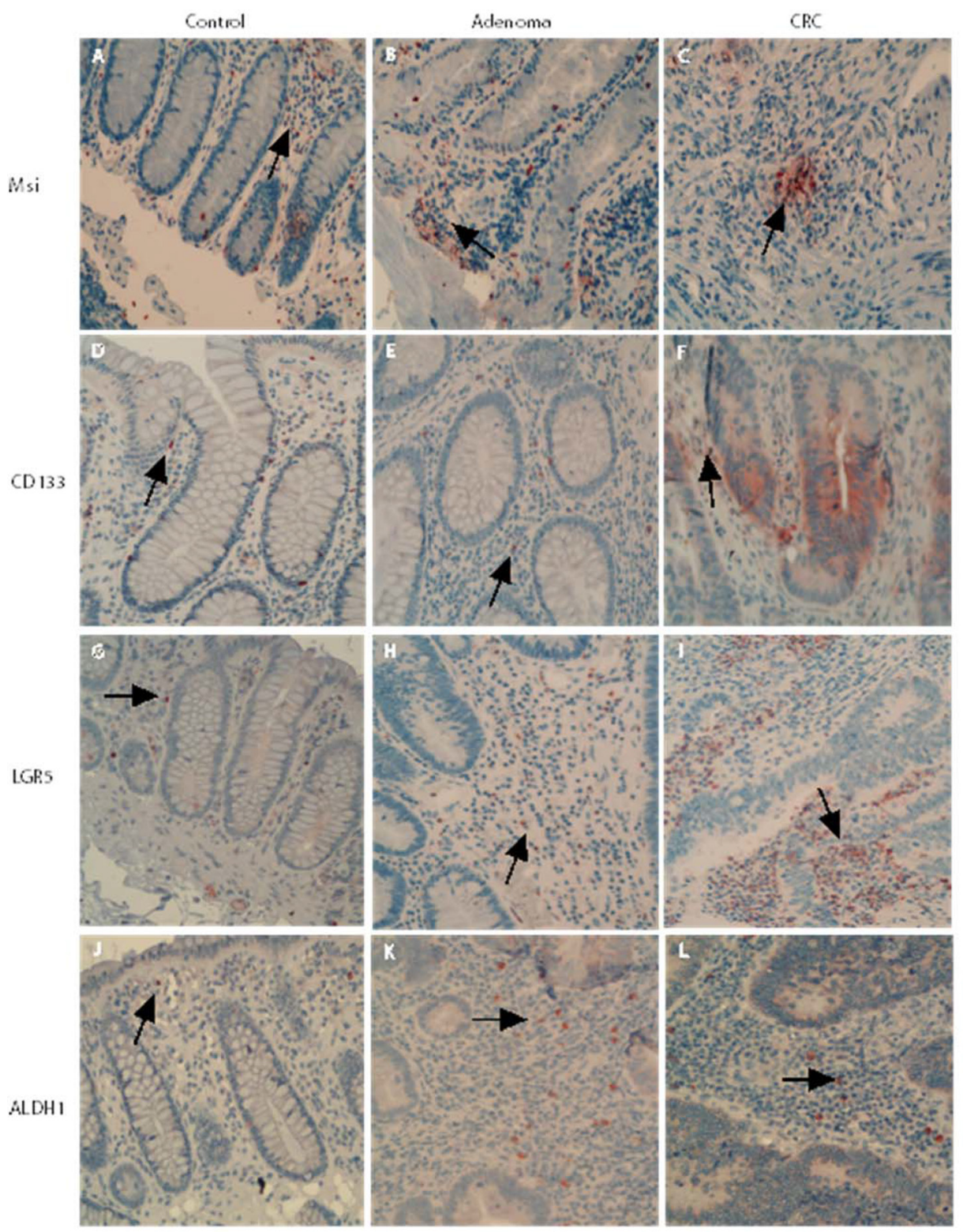

Figure 3: Mesenchymal stem-like markers in the adenomatous/cancerous tumor stroma. The stromal cells positive for stem-like markers were diffusely distributed and the numbers of cells positive for stem-like markers Msi, CD133, LGR5 and ALDH1 in normal lamina propria and the densities were higher than that in the crypt (Figure 3A, 3D, 3G and 3J). In both the adenomas and CRC stroma, the cells positive for stem-like markers were increased and diffusely distributed in the tumor stroma between adenomatous (Figure 3B, 3E, 3H and 3J) or CRC (Figure 3C, 3F, 3I and 3L) epithelium. 
Because fibroblasts are the main type of stromal cells and the activation of stromal fibroblasts are involved in the initiation and progression of CRC [23, 24], we examined the expression of fibroblasts labeled with vimentin with $\mathrm{IHC}$ and the proliferative activity of vimentin positive fibroblasts with double IHC in the adenoma/CRC stroma. The results of vimentin IHC showed that fibroblasts were observed in the whole stroma within both the pericryptal and non-pericryptal regions in adenoma, $\mathrm{CRC}$ and control group sections (Figure 5D-5F). In the adenoma section, dense fibroblasts were observed in the whole stroma but were particularly dense in the peri-adenomatous epithelium region (Figure $5 \mathrm{E}$ ) compared to controls (Figure 5D). In the CRC sections, the presentation of fibroblasts was similar to that of the adenomas and many fibroblasts could be found in the tumor stroma (Figure 5F). When the density of vimentin positive fibroblasts was graded, the grading scores were higher in adenomas (Figure $5 \mathrm{H}$, grey bar) and CRCs (Figure 5H, black bar) relative to controls (Figure 5H, white bar), although statistical significance was not reached.

The results of double IHC demonstrated that many of the PCNA positive cells in the control (Figure 6A), adenoma (Figure 6B) and CRC (Figure 6C) stromal cells were vimentin positive fibroblasts and confirmed that vimentin positive fibroblasts in the adenoma/CRC stroma might have a high proliferative capacity.
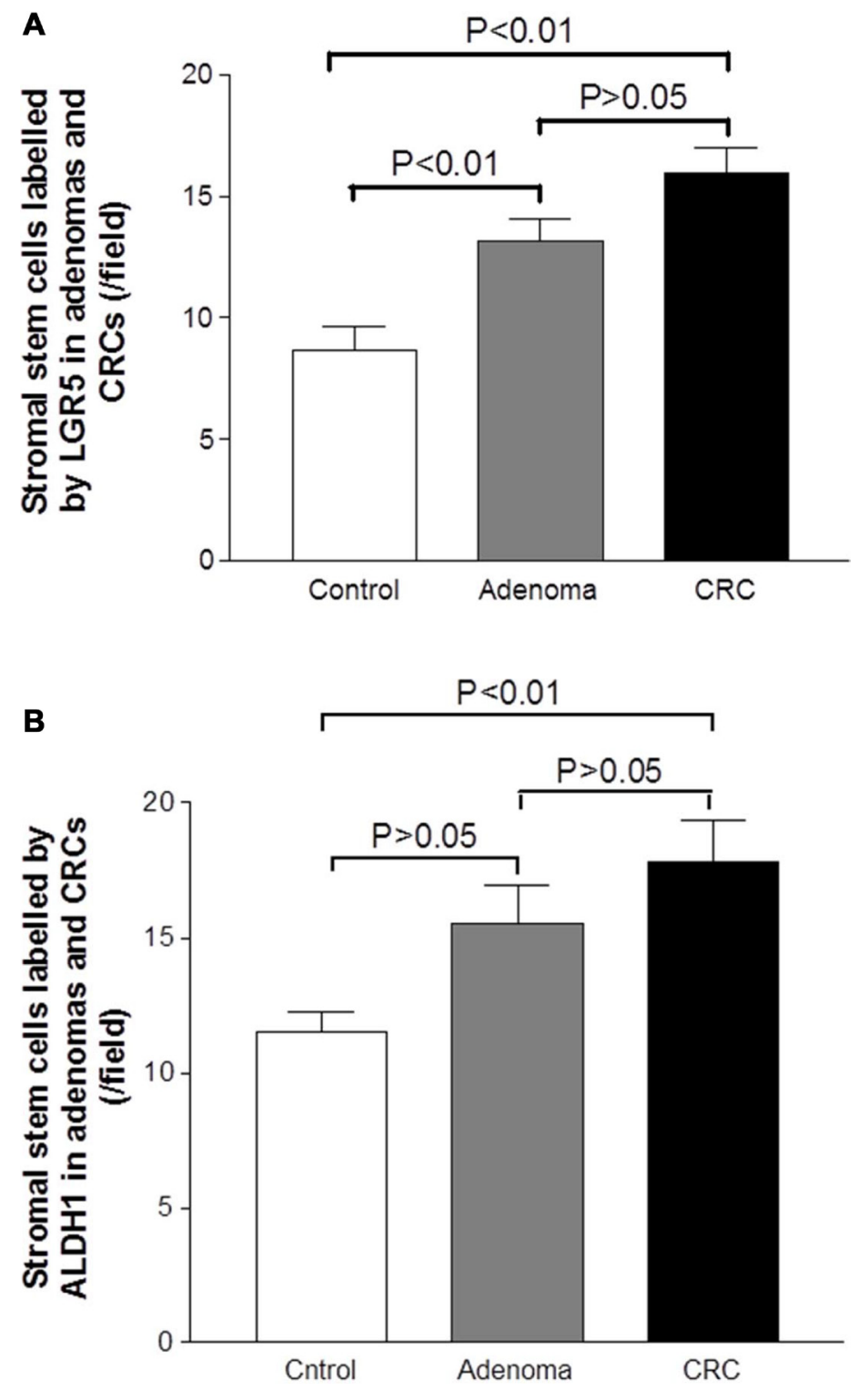

Figure 4: Graphic analysis of the density of mesenchymal stem-like cells labeled by LGR5 and ALDH1 in the adenomatous/cancerous tumor stroma. The densities of mesenchymal stem-like cells labeled by LGR5 (Figure 4A) and ALDH1 (Figure 4B) were increased in the adenoma and CRC sections relative with the control section, that began from the adenoma lesion and even higher in the CRC lesion. 


\section{DISCUSSION}

In this study, we examined the presentation pattern of stem-like markers in different stages and compartments of the adenoma-carcinoma sequence and translational crypts adjacent to adenomas and CRCs. We were able to demonstrate an altered localization and expression pattern of cells positive for stem-like markers in those tissues. In addition, we have also observed an increase in cells positive for stem-like markers in the adenoma/CRC stroma that accompanied by an active proliferation rate in the tumor stromal cells, and many of the proliferative stromal cells were identified as vimentin positive fibroblasts. These findings suggest that the change in the presentation pattern of the stem-like markers in different stages and compartments of the adenoma-carcinoma sequence reflects an ongoing tumorigenesis. Such change might be one of the hallmarks of colorectal tumorigenesis and related to the stromal response that resulted from tumorigenesis.

Under physiological conditions, only a low density of cells positive for stem-like markers reside in the base of crypts, and they play an important role in maintaining normal
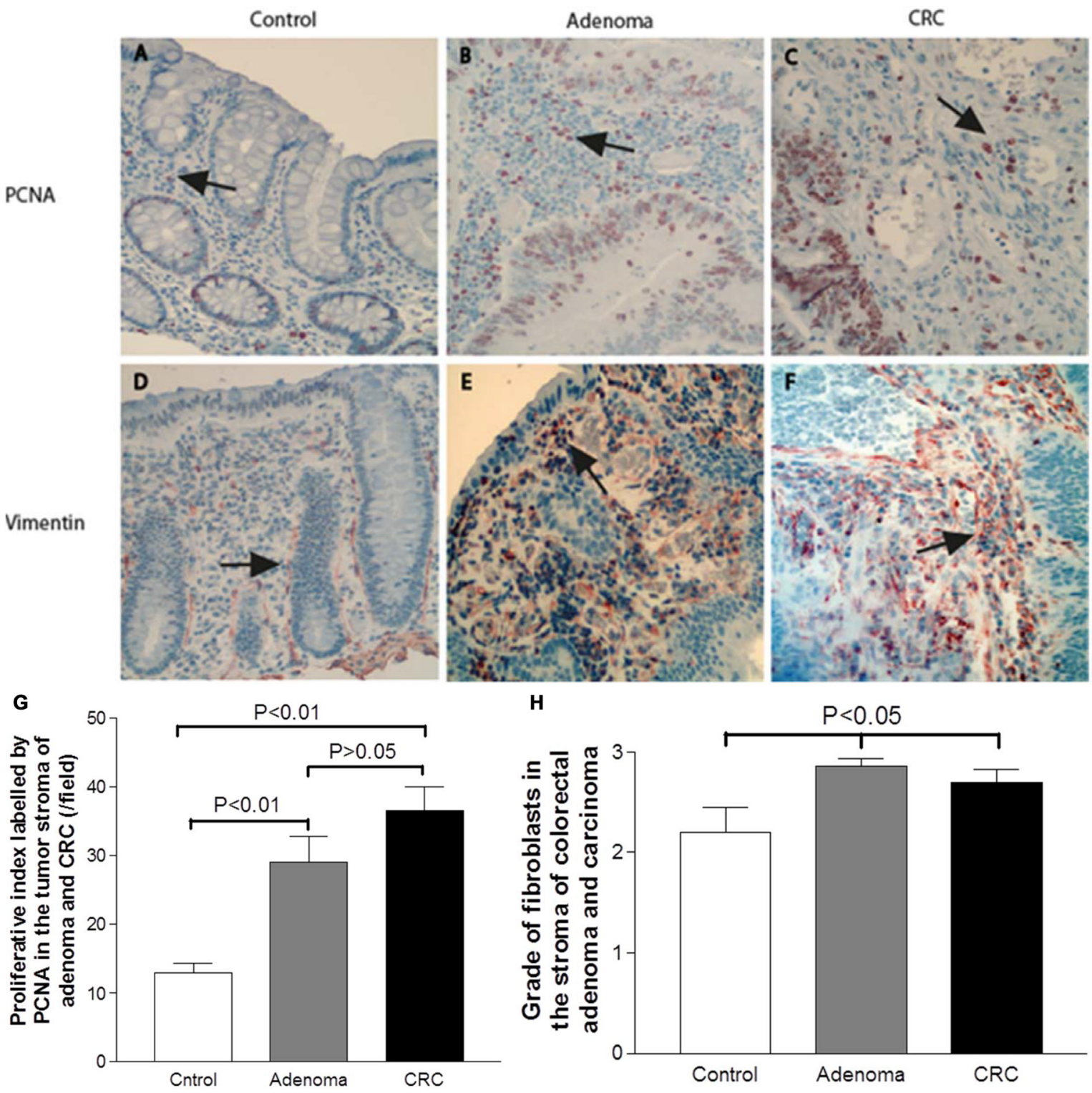

Figure 5: Increased proliferation rate of stromal cells that primarily identified as vimentin positive fibroblasts in adenomas and CRCs. The proliferation rate in the tumor stromal cells was evaluated with PCNA IHC (Figure 5A-5C). The stromal cells positive for PCNA in both the adenoma and the CRC were significantly increased relative to the control (Figure 5G) (both $P<0.0001$ ). Further IHC examination revealed that fibroblasts labeled by vimentin were observed within both the pericryptal and non-pericryptal regions in the stroma of control (Figure 5D), adenoma (Figure 5E) and CRC (Figure 5F). When the density of fibroblasts was graded, the grading scores were shown in a non-statistically increased in the adenoma (Figure $\mathbf{5 H}$, grey bar) and CRC stroma (Figure 5H, black bar) relative to the control (Figure $5 \mathrm{H}$, white bar). 
tissue homeostasis and function. Thus, it is not unusual that the number of cells positive for stem-like markers in the normal crypt and lamina propria was low in control tissues. However, when colorectal mucosa becomes tumorigenic, the stem-like cells are activated and the number of cells positive for stem-like markers was significantly increased. In the current study, we observed a large change in the presentation of stem-like markers in different compartments of adenoma and CRC tissues. The cells positive for various types of stem-like markers were observed in both the adenomatous/ cancerous epithelium and the tumor stroma. The cells were mostly distributed in a patchy pattern in the adenomatous/ cancerous epithelium. This finding might reflect the fact that stem cells are substantially activated during the procession of colorectal tumorigenesis.

The importance of stem cells in the initiating of adenomas has been recognized $[2,5,11]$. Several potential stem-like markers have been used to identify stem cells in adenomas/CRC. LGR5 and ALDH1 are the two most promising and established stem-like markers and have been frequently used [3, 5, 7, 10, 15]. Studies have reported that LGR5 and ALDH1 expression can reflect the changes in stem-like cells during the process of colorectal tumorigenesis. However, whether the mechanism is topdown or bottom-up is a matter of debate $[11,16]$. Some studies suggest that dysplastic cells are routinely found at the luminal surface of adenomatous crypts that contain genetic alterations in the adenomatous polyposis coli (APC) gene and neoplasia-associated patterns of gene expression. Stem-like cells are also observed at the luminal surface of adenomatous crypts. Thus, these findings imply that the development of adenomatous polyps proceeds through a top-down mechanism. Alternatively, genetically-altered cells in the superficial portions of the mucosae spread laterally and downward to form new crypts that first connect to preexisting normal crypts and eventually replace them [17]. However, evidence from other studies has supported the idea that colorectal polyps are polyclonal and arise from a combination of host genetic features via a bottom-up mechanism [18, 19]. The transitional mucosa is characterized morphologically by an increase in mucosal thickness, lengthening of the crypts and goblet cell hyperplasia. Since transitional mucosa reflects the ongoing histopathological procession of tumorigenesis, studying the changes of pathological aspects inside crypts might provide critical information for the understanding of tumorigenesis procession. Indeed, several groups including ours have demonstrated the histological, genetic, histochemical and immunological changes in the mucosa adjacent to the adenoma and CRC epithelium [20-25]. However, there is very limited information available regarding the change of stem-like cells in the transitional mucosa during the development of CRC. To our best knowledge, the current study is the first of its kind to demonstrate the temporal and spatial changes of stem-like markers in the transitional mucosa from colorectal adenomas to sporadic CRCs. We have observed an altered presentation pattern of cells positive for stem-like markers in the transitional mucosa close to adenomas and CRCs. In controls, cells positive to stemlike markers were only located at the base of the crypts. However, some of positive cells were located in the middle part and occasionally expanded to the top of the transitional crypt close to adenomas and CRCs. In light of our results, such altered location of cells positive for stem-like markers in the transitional mucosa may reflect the undergoing expanding path of stem-like cells inside preneoplasic crypts and could be the supportive evidence for the bottom-up hypothesis. This information could also be very useful as an indicator of a malignancy potential and as a target for novel therapeutic approaches.

With respect to the relationship between stemlike cells (labeled with LGR 5 and ALDH1) and clinicopathological parameters of patients with adenomas

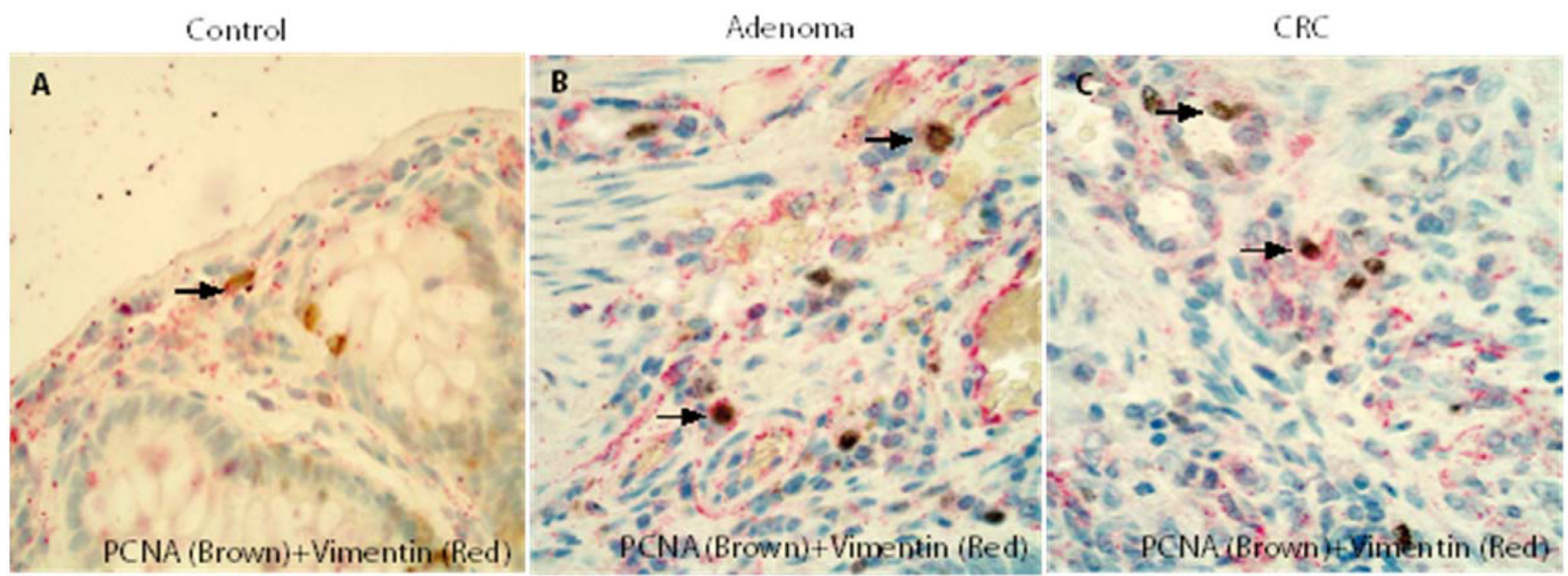

Figure 6: Double IHCs confirmed that fibroblasts in the adenoma/CRC stroma might have a high proliferative capacity. The results of double IHC demonstrated that many of PCNA positive stromal cells in the control (Figure 6A), adenomas (Figure 6B) and CRCs (Figure 6C) were fibroblasts. 
and CRCs, we have observed slight increased scores of LGR5 and ALDH1 positive cells in the adenomatous epithelium with high degree of dysplasia. We have also found that grading scores of LGR5 and ALDH1 positive cells in the cancerous epithelium were significantly increased in CRC patients with advanced TNM stages than those with early stages. Those observations are consist with other groups' findings [15, 26-32], and imply that increased stem-like cell density might be the potential predicators for disease stage progression in both the adenoma and CRC. Mesenchymal stem like cell can be observed in the tumor stroma, it plays a critical role in remodeling tumor stroma and forming a supportive environment for cancer growth and metastasis [33]. In this study, many mesenchymal cells positive for these potential stem-like markers could be observed in the adenoma/CRC stroma, which was accompanied by an increase in proliferation in the stromal cells that has been previously reported by our group [34]. When the cell positive for stem-like markers was analyzed against the clinical pathological parameters in the adenomatous/ cancerous stroma, we have observed that the grading score of cell positive for LGR5 and ALDH1 was not associated with most clinical pathological parameters i.e. degree of dysplasia and histological types in the adenoma and TNM stage in the CRC. However, the density grading scores of positive cell were higher in CRC patients with node involvement than those without, although the difference did not reach statistical significance. This observation suggested that the tumorigenesis may results in a general stem-like cell response in the tumor stroma, such activation might contribute to the diseases progression.

Since vimentin-expression is one of the hallmarks of epithelial-mesenchymal-transition (EMT) during the progression of $\mathrm{CRC}$, we have therefore further examined the activity of vimentin positive fibroblasts in the adenomatous/cancerous stroma. The results showed that these PCNA positive proliferative stromal cells were mostly identified as vimentin positive fibroblasts in the adenoma/CRC stroma. As illustrated by many studies [1, 34-36], fibroblasts are the main type of stromal cells in adenoma and CRC tissue and they promote the progression of adenoma to CRC. The exact mechanisms for the elevated proliferation seen in tumor stromal fibroblasts are thus far undetermined. It has been reported that vimentin-expression is one of the hallmarks of EMT during the progression of CRC and vimentin positive stromal cells may be with tumorigenic cell characteristics and play a vital role during the invasion of CRC. In addition, many factors are related to the stromal activation during the development of human cancers $[14,35]$. For instance, the release of cytokines from the tumor microenvironment may participate in the modulation of the proliferative activity of the stromal cell [37]. In our previous studies, we have demonstrated an elevated expression pattern of IL-17A in the tumor microenvironment of adenoma/CRC [25, 38, 39]. It has been found that IL-17A stimulates stem-like cells and promote the development of various types of cancers [40-43]. Our current findings may provide further evidence to support the notion that the activation of vimentin positive fibroblasts in the adenomatous/cancerous stroma might be one of the hallmarks of EMT and are involved in the process of colorectal tumorigenesis.

In summary, this study showed a temporal and spatial variation of stem-like markers in different compartments and stages of the colorectal adenoma-carcinoma sequence, including adenoma/CRC epithelium, transitional crypts and tumor stroma, throughout all stages of the colorectal adenoma-carcinoma sequence. Such dynamic change of stem-like markers reflect the ongoing tumorigenesis and may be a hallmark of the adenoma-carcinoma transition.

\section{MATERIALS AND METHODS}

A total of 30 biopsies of colorectal adenomas excised completely by endoscopic polypectomy (male/female ratio 22/8; age 36-70 years) and 30 biopsies of CRC excised by surgery (male/female ratio 17/13; age 49-70 years) were included in this study. No patients received radiotherapy and/or chemotherapy preoperatively. Moreover, biopsies from 12 subjects (male 8, female 4, ages 35-60 years) with normal colonoscopy and histology served as a normal control group. All biopsies were prepared and embedded in paraffin routinely. Sections were cut at a thickness of $4 \mu \mathrm{m}$ and then stained with hematoxylin and eosin $(H \& E)$. Basic information was summarized in Table 1. This study was approved by the local Medical Research Committee.

\section{Immunohistochemistry (IHC) for stem-like markers, stromal proliferative activity labeled by PCNA and stromal fibroblasts labeled by vimentin}

Sections for IHC were deparaffinized in xylene, rehydrated in graded ethanol, and incubated in a $0.3 \%$ $\mathrm{H}_{2} \mathrm{O}_{2}$ solution in methanol for 15 minutes to block endogenous peroxidases. Antigen retrieval was achieved by boiling sections for 15 minutes in $0.01 \mathrm{M}$ citrate buffer, pH 6.0. Nonspecific binding was blocked by incubating sections in phosphate buffered saline (PBS) containing $4 \%$ normal bovine serum and $0.25 \%$ Triton-X 100. The slides were rinsed three times with PBS with $0.25 \%$ Triton-X 100 (PBS-T) for 5 min and incubated overnight at $4{ }^{\circ} \mathrm{C}$ with different antibodies for putative stem-like markers: anti-Msi (DAKO, Carpinteria, CA, USA), anti-CD133 (Abcam, Cambridge, UK), antiLGR5 (MBL International, Woburn, MA 01801, USA) and anti-ALDH1 (BD Bioscience, San Jose, CA, USA) individually. For PCNA and vimentin IHC, sections were incubated overnight at $4{ }^{\circ} \mathrm{C}$ with primary antibodies: antiPCNA polyclonal antibody (Abcam, Cambridge, UK) and 
Table 1: Basic histological information of patients and normal individuals

\begin{tabular}{|c|c|c|c|c|c|c|c|c|}
\hline & \multirow[t]{2}{*}{$N$} & \multicolumn{2}{|c|}{ Location } & \multicolumn{2}{|c|}{ Histopathology } & \multicolumn{3}{|c|}{ Dysplasia degree } \\
\hline & & colon & rectum & tubular & tubulovillous & LGD & & HGD \\
\hline Normal & 12 & 5 & 7 & & & & & \\
\hline \multirow[t]{3}{*}{ Adenoma } & 30 & 20 & 10 & 20 & 10 & 18 & & 12 \\
\hline & & & & & & \multicolumn{3}{|c|}{ TNM stage } \\
\hline & & & & \multicolumn{2}{|c|}{ adenocarcinoma } & $\mathrm{I}$ & II & III \\
\hline $\mathrm{CRC}$ & 30 & 16 & 14 & \multicolumn{2}{|c|}{30} & 7 & 10 & 13 \\
\hline
\end{tabular}

LGD: low grading of dysplasia.

HGD: high grading of dysplasia.

anti-Vimentin monoclonal antibody (DAKO, Carpinteria, CA, USA), respectively. After primary antibody staining, the slides were washed with PBS-T for 10 minutes, and detection was performed using the Vectastain Elite $A B C$ Kit (Vector Lab., Burlingame, CA, USA) according to the manufacturer's instructions and our published method $[14,15]$. Next, 3-Amino-9-ethylcarbazole (AEC; Vector Laboratories, Burlingame, CA, USA) was used as the chromogen, and slides were counterstained with Mayer's hematoxylin. Negative control slides for IHC were made routinely: (1) primary antibodies were substituted with the isotype-matched control antibodies; and (2) a secondary antibody was substituted with PBS-T.

\section{Double IHC for the examination of proliferative activity in stromal fibroblasts}

Because fibroblasts are the main type of tumor stromal cells [44], we examined the proliferative activity of fibroblasts with double IHC in the tumor stroma. Double IHC with PCNA/ Vimentin (to label fibroblasts) antibodies was performed using the EnVision Doublestain System kit (DAKO, Carpinteria, CA, USA) according to the manufacturer's instructions and our published methods $[45,46]$. In brief, the slides were incubated overnight at $4^{\circ} \mathrm{C}$ with anti-PCNA antibody after antigen retrieval and then incubated with labeled polymer-horseradish peroxidase-anti-mouse and anti-rabbit antibodies for 30 minutes at room temperature. Peroxidase activity was detected with the enzyme substrate 3,3'-diaminobenzidine tetrachloride $(D A B)$. After quenching the enzyme reaction, the slides were incubated in Doublestain Block at room temperature for 5 minutes to block endogenous phosphatases. The slides were then incubated with anti-Vimentin antibody for 2 hours at room temperature. After washing, the slides were incubated with labeled polymer-alkaline phosphatase anti-mouse and antirabbit antibodies for 30 minutes at room temperature. A Fast Red chromogen substrate solution was used to visualize antivimentin antibody. The sections were slightly counterstained with Mayer's hematoxylin.

\section{Morphological evaluation}

The semi-quantified density grading of stem-like positive cells was employed according to the method described in our previous publication [45, 47]. In brief, semi-quantitative scoring was conducted in at least five wellorientated fields with abundant positive cell distribution from each slide under $400 \times$ high-power magnification. In the adenoma/CRC epithelium, the number of LGR5 positive and ALDH1 positive cells was graded on a scale of $0-3$ : grade 0 indicated an absence of LGR5/ALDH1 positive cells; grade 1 indicated a $1 \%-5 \%$ presence of LGR5/ALDH1 positive cells; grade 2 indicated a $6 \%-25 \%$ presence of LGR5/ALDH1 positive cells; and grade 3 indicated a $26 \%-100 \%$ presence of LGR $5 /$ ALDH1 positive cells. The results were grouped as positive (grade 2 or 3 ), or negative (grade 0 or 1 ), given that the normal gastric mucosa was identified as grade 1 for LGR5/ALDH1 expression [48]. In tumor stroma, fibroblasts (labeled by Vimentin) were found at a very high density and were evaluated with light microscopy using the following criteria described by Adegboyega et al. [49]: negative immunoreactivity (IR) $(-), 1 \%$ to $25 \%$ positive cells $(+), 25 \%$ to $50 \%$ positive cells $(++)$, and greater than $50 \%$ positive cells $(+++)$. The number of PCNA positive (nucleus) cells in the tumor stroma were counted in at least 5 optional fields with abundant distribution from each slide under a $400 \times$ high-power magnification. The average values were used for statistical analysis.

\section{Statistical analysis}

The results were expressed as the mean \pm SEM unless otherwise stated. Statistical significance was evaluated by the Mann-Whitney and Kruskal-Wallis tests. Values of $P<0.05$ were considered significant.

\section{CONFLICTS OF INTEREST}

All authors declare no conflicts of interest.

\section{FUNDING}

This study was funded by Innovation Scientists and Technicians Troop Construction Projects of Henan Province (C20150009) to Cui G and National Nature Science Foundation of China (81071969) to Cui G. 


\section{REFERENCES}

1. Almholt K, Johnsen M. Stromal cell involvement in cancer. Recent Results Cancer Res. 2003; 162:31-42.

2. Barker N, Ridgway RA, van Es JH, van de Wetering M, Begthel H, van den Born M, Danenberg E, Clarke AR, Sansom OJ, Clevers H. Crypt stem cells as the cells-oforigin of intestinal cancer. Nature. 2009; 457:608-611.

3. Zhou F, Mu YD, Liang J, Liu ZX, Zhou D, Ning WL, Li YZ, Ding D, Zhang JF. Aldehyde dehydrogenase 1: a specific cancer stem cell marker for human colorectal carcinoma. Mol Med Rep. 2015; 11:3894-3899.

4. Todaro M, Francipane MG, Medema JP, Stassi G. Colon cancer stem cells: promise of targeted therapy. Gastroenterology. 2010; 138:2151-2162.

5. Baker AM, Graham TA, Elia G, Wright NA, RodriguezJusto M. Characterization of LGR5 stem cells in colorectal adenomas and carcinomas. Sci Rep. 2015; 5:8654.

6. Kozovska Z, Gabrisova V, Kucerova L. Colon cancer: cancer stem cells markers, drug resistance and treatment. Biomed Pharmacother. 2014; 68:911-916.

7. Rassouli FB, Matin MM, Saeinasab M. Cancer stem cells in human digestive tract malignancies. Tumour Biol. 2016; $37: 7-21$

8. Hong I, Hong SW, Chang YG, Lee WY, Lee B, Kang YK, Kim YS, Paik IW, Lee H. Expression of the Cancer Stem Cell Markers CD44 and CD133 in Colorectal Cancer: An Immunohistochemical Staining Analysis. Ann Coloproctol. 2015; 31:84-91.

9. Fan LF, Dong WG, Jiang CQ, Xia D, Liao F, Yu QF. Expression of putative stem cell genes Musashi-1 and betal-integrin in human colorectal adenomas and adenocarcinomas. Int J Colorectal Dis. 2010; 25:17-23.

10. Jang BG, Kim HS, Kim KJ, Rhee YY, Kim WH, Kang GH. Distribution of intestinal stem cell markers in colorectal precancerous lesions. Histopathology. 2016; 68:567-577.

11. Leedham SJ, Wright NA. Expansion of a mutated clone: from stem cell to tumour. J Clin Pathol. 2008; 61:164-171.

12. Quante M, Wang TC. Stem cells in gastroenterology and hepatology. Nat Rev Gastroenterol Hepatol. 2009; 6:724-737.

13. Chen L, Shen R, Ye Y, Pu XA, Liu X, Duan W, Wen J, Zimmerer J, Wang Y, Liu Y, Lasky LC, Heerema NA, Perrotti D, et al. Precancerous stem cells have the potential for both benign and malignant differentiation. PLoS One. 2007; 2:e293.

14. Pavon LF, Sibov TT, de Oliveira DM, Marti LC, Cabral FR, de Souza JG, Boufleur P, Malheiros SM, de Paiva Neto MA, da Cruz EF, Chudzinski-Tavassi AM, Cavalheiro S. Mesenchymal stem cell-like properties of CD133+ glioblastoma initiating cells. Oncotarget. 2016; 7: 40546-40557. doi: 10.18632/oncotarget.9658.

15. Bartley AN, Parikh N, Hsu CH, Roe DJ, Buckmeier JA, Corley L, Phipps RA, Gallick G, Lance P, Thompson PA, Hamilton SR. Colorectal adenoma stem-like cell populations: associations with adenoma characteristics and metachronous colorectal neoplasia. Cancer Prev Res (Phila). 2013; 6:1162-1170.

16. Wright NA, Poulsom R. Top down or bottom up? Competing management structures in the morphogenesis of colorectal neoplasms. Gut. 2002; 51:306-308.

17. Shih IM, Wang TL, Traverso G, Romans K, Hamilton SR, Ben-Sasson S, Kinzler KW, Vogelstein B. Top-down morphogenesis of colorectal tumors. Proc Natl Acad Sci USA. 2001; 98:2640-2645.

18. Preston SL, Wong WM, Chan AO, Poulsom R, Jeffery R, Goodlad RA, Mandir N, Elia G, Novelli M, Bodmer WF, Tomlinson IP, Wright NA. Bottom-up histogenesis of colorectal adenomas: origin in the monocryptal adenoma and initial expansion by crypt fission. Cancer Res. 2003; 63:3819-3825.

19. Thirlwell C, Will OC, Domingo E, Graham TA, McDonald SA, Oukrif D, Jeffrey R, Gorman M, Rodriguez-Justo M, ChinAleong J, Clark SK, Novelli MR, Jankowski JA, et al. Clonality assessment and clonal ordering of individual neoplastic crypts shows polyclonality of colorectal adenomas. Gastroenterology. 2010; 138:1441-1454.

20. Zhang H, Ahmadi A, Arbman G, Zdolsek J, Carstensen J, Nordenskjold B, Soderkvist P, Sun XF. Glutathione S-transferase T1 and M1 genotypes in normal mucosa, transitional mucosa and colorectal adenocarcinoma. Int $\mathrm{J}$ Cancer. 1999; 84:135-138.

21. Giuffre G, Barresi G, Sturniolo GC, Sarnelli R, D'Inca R, Tuccari G. Immunohistochemical expression of metallothionein in normal human colorectal mucosa, in adenomas and in adenocarcinomas and their associated metastases. Histopathology. 1996; 29:347-354.

22. Zhang H, Nordenskjold B, Dufmats M, Soderkvist P, Sun XF. K-ras mutations in colorectal adenocarcinomas and neighbouring transitional mucosa. Eur J Cancer. 1998; 34:2053-2057.

23. Tamai O, Miyazato H, Shiraishi M, Kusano T, Muto Y. Morphologic and mucin histochemical analysis of transitional zones in advanced ulcerated colorectal carcinomas: potential prognostic indicators. J Surg Oncol. 1998; 67:85-89.

24. Cui G, Yuan A, Goll R, Olsen T, Husebekk A, Vonen B, Florholmen J. Distinct changes of dendritic cell number and IL-12 mRNA level in adjacent mucosa throughout the colorectal adenoma-carcinoma sequence. Cancer Immunol Immunother. 2007; 56:1993-2001.

25. Cui G, Yang H, Zhao J, Yuan A, Florholmen J. Elevated proinflammatory cytokine IL-17A in the adjacent tissues along the adenoma-carcinoma sequence. Pathol Oncol Res. $2015 ; 21: 139-146$.

26. Wu W, Cao J, Ji Z, Wang J, Jiang T, Ding H. Co-expression of Lgr5 and CXCR4 characterizes cancer stem-like cells of colorectal cancer. Oncotarget. 2016; 7:81144-81155. doi: 10.18632/oncotarget.13214.

27. Galizia G, Gemei M, Del Vecchio L, Zamboli A, Di Noto R, Mirabelli P, Salvatore F, Castellano P, Orditura M, De 
Vita F, Pinto M, Pignatelli C, Lieto E. Combined CD133/ CD44 expression as a prognostic indicator of disease-free survival in patients with colorectal cancer. Arch Surg. 2012; 147:18-24.

28. Liu D, Sun J, Zhu J, Zhou H, Zhang X, Zhang Y. Expression and clinical significance of colorectal cancer stem cell marker EpCAMhigh/CD44+ in colorectal cancer. Oncology letters. 2014; 7:1544-1548.

29. He S, Zhou H, Zhu X, Hu S, Fei M, Wan D, Gu W, Yang X, Shi D, Zhou J, Zhou J, Zhu Z, Wang L, et al. Expression of Lgr5, a marker of intestinal stem cells, in colorectal cancer and its clinicopathological significance. Biomed Pharmacother. 2014; 68:507-513.

30. Ong CW, Chong PY, McArt DG, Chan JY, Tan HT, Kumar AP, Chung MC, Clement MV, Soong R, Van Schaeybroeck S, Waugh DJ, Johnston PG, Dunne PD, Salto-Tellez M. The prognostic value of the stem-like group in colorectal cancer using a panel of immunohistochemistry markers. Oncotarget. 2015; 6:12763-12773. doi: 10.18632/ oncotarget.3497.

31. Becker L, Huang Q, Mashimo H. Immunostaining of Lgr5, an intestinal stem cell marker, in normal and premalignant human gastrointestinal tissue. Sci World J. 2008; 8:1168-1176.

32. Takeda K, Kinoshita I, Shimizu Y, Matsuno Y, Shichinohe T, Dosaka-Akita H. Expression of LGR5, an intestinal stem cell marker, during each stage of colorectal tumorigenesis. Anticancer Res. 2011; 31:263-270.

33. Wu XB, Liu Y, Wang GH, Xu X, Cai Y, Wang HY, Li YQ, Meng HF, Dai F, Jin JD. Mesenchymal stem cells promote colorectal cancer progression through AMPK/mTORmediated NF-kappaB activation. Sci Rep. 2016; 6:21420.

34. Cui G, Yuan A, Vonen B, Florholmen J. Progressive cellular response in the lamina propria of the colorectal adenomacarcinoma sequence. Histopathology. 2009; 54:550-560.

35. Bhowmick NA, Neilson EG, Moses HL. Stromal fibroblasts in cancer initiation and progression. Nature. 2004; 432:332-337.

36. De Wever O, Mareel M. Role of tissue stroma in cancer cell invasion. J Pathol. 2003; 200:429-447.

37. McLean MH, Murray GI, Stewart KN, Norrie G, Mayer C, Hold GL, Thomson J, Fyfe N, Hope M, Mowat NA, Drew JE, El-Omar EM. The inflammatory microenvironment in colorectal neoplasia. PLoS One. 2011; 6:e15366.

38. Cui G, Yuan A, Goll R, Florholmen J. IL-17A in the tumor microenvironment of the human colorectal adenoma-carcinoma sequence. Scand J Gastroenterol. 2012; 47:1304-1312.

39. Qi H, Yang H, Xu G, Ren J, Hua W, Shi Y, Torsvik M, Florholmen J, Cui G. Therapeutic efficacy of IL-17A antibody injection in preventing the development of colitis associated carcinogenesis in mice. Immunobiology. 2015; 220:54-59.
40. Yang S, Wang B, Guan C, Wu B, Cai C, Wang M, Zhang B, Liu T, Yang P. Foxp3+IL-17+ T cells promote development of cancer-initiating cells in colorectal cancer. J Leukoc Biol. 2011; 89:85-91.

41. Zhang Q, Yamaza T, Kelly AP, Shi S, Wang S, Brown J, Wang L, French SW, Shi S, Le AD. Tumor-like stem cells derived from human keloid are governed by the inflammatory niche driven by IL-17/IL-6 axis. PLoS One. 2009; 4:e7798.

42. Charles KA, Kulbe H, Soper R, Escorcio-Correia M, Lawrence T, Schultheis A, Chakravarty P, Thompson RG, Kollias G, Smyth JF, Balkwill FR, Hagemann T. The tumorpromoting actions of TNF-alpha involve TNFR1 and IL-17 in ovarian cancer in mice and humans. J Clin Invest. 2009; 119:3011-3023.

43. Nam JS, Terabe M, Kang MJ, Chae H, Voong N, Yang YA, Laurence A, Michalowska A, Mamura M, Lonning S, Berzofsky JA, Wakefield LM. Transforming growth factor beta subverts the immune system into directly promoting tumor growth through interleukin-17. Cancer Res. 2008; 68:3915-3923.

44. Ngan CY, Yamamoto H, Seshimo I, Tsujino T, Man-i M, Ikeda JI, Konishi K, Takemasa I, Ikeda M, Sekimoto M, Matsuura N, Monden M. Quantitative evaluation of vimentin expression in tumour stroma of colorectal cancer. Br J Cancer. 2007; 96:986-992.

45. Cui G, Qi H, Gundersen MD, Yang H, Christiansen I, Sorbye SW, Goll R, Florholmen J. Dynamics of the IL33/ST2 network in the progression of human colorectal adenoma to sporadic colorectal cancer. Cancer Immunol Immunother. 2015; 64:181-190.

46. Hua W, Yuan A, Zheng W, Li C, Cui J, Pang Z, Zhang L, Li Z, Goll R, Cui G. Accumulation of FoxP3+ T regulatory cells in the tumor microenvironment of human colorectal adenomas. Pathol Res Pract. 2016; 212:106-112.

47. Cui G, Goll R, Olsen T, Steigen SE, Husebekk A, Vonen B, Florholmen J. Reduced expression of microenvironmental Th1 cytokines accompanies adenomas-carcinomas sequence of colorectum. Cancer Immunol Immunother. 2007; 56:985-995.

48. Jang BG, Lee BL, Kim WH. Distribution of LGR5+ cells and associated implications during the early stage of gastric tumorigenesis. PLoS One. 2013; 8:e82390.

49. Adegboyega PA, Ololade O, Saada J, Mifflin R, Di Mari JF, Powell DW. Subepithelial myofibroblasts express cyclooxygenase-2 in colorectal tubular adenomas. Clin Cancer Res. 2004; 10:5870-5879. 